\title{
Extension of Improved Particle and Energy Confinement Regime in the Core of LHD Plasma*)
}

\author{
Osamu KANEKO, Masayuki YOKOYAMA, Mikiro YOSHINUMA, Ken-ichi NAGAOKA,
}

Katsumi IDA, Ryuichi SAKAMOTO, Tsuyoshi AKIYAMA, Naoko ASHIKAWA,

Hirotaka CHIKARAISHI, Masahiko EMOTO, Hisamichi FUNABA, Pavel GONCHAROV, Motoshi GOTO, Takuya GOTO, Shinji HAMAGUCHI, Katsuji ICHIGUCHI, Takeshi IDO, Hiroe IGAMI, Katsunori IKEDA, Shinsaku IMAGAWA, Mitsutaka ISOBE, Kimitaka ITOH, Akifumi IWAMOTO, Ryutaro KANNO, Hiroshi KASAHARA, Naohiro KASUYA, Daiji KATO,

Kazuo KAWAHATA, Masahiro KOBAYASHI, Shin KUBO, Ryuhei KUMAZAWA,

Ryuji MAEKAWA, Suguru MASUZAKI, Toshiyuki MITO, Jun-ichi MIYAZAWA,

Naoki MIZUGUCHI, Tomohiro MORISAKI, Shigeru MORITA, Gen MOTOJIMA,

Sadatsugu MUTO, Takashi MUTOH, Yoshio NAGAYAMA, Noriyoshi NAKAJIMA, Yukio NAKAMURA, Hideya NAKANISHI, Kazumichi NARIHARA, Yoshiro NARUSHIMA,

Kiyohiko NISHIMURA, Masaki NISHIURA, Tetsuhiro OBANA, Satoshi OHDACHI, Nobuyoshi OHYABU, Masaki OSAKABE, Tetsuo OZAKI, Byron J. PETERSON, Akio SAGARA, Kenji SAITO, Satoru SAKAKIBARA, Shinsuke SATAKE, Kuninori SATO, Tetsuo SEKI, Akihiro SHIMIZU, Takashi SHIMOZUMA, Mamoru SHOJI, Shigeru SUDO, Hideo SUGAMA, Chihiro SUZUKI, Yasuhiro SUZUKI, Hiromi TAKAHASHI, Kazuya TAKAHATA, Yasuhiko TAKEIRI, Hitoshi TAMURA, Naoki TAMURA, Kenji TANAKA, Shinichiro TODA, Kazuo TOI, Masayuki TOKITANI, Tokihiko TOKUZAWA, Katsuyoshi TSUMORI, Kiyomasa WATANABE, Ichihiro YAMADA, Hiroshi YAMADA, Osamu YAMAGISHI,

Nagato YANAGI, Shinji YOSHIMURA, Yasuo YOSHIMURA and Akio KOMORI National Institute for Fusion Science, Toki 509-5292 Japan

(Received 1 September 2008 / Accepted 23 April 2009)

\begin{abstract}
Recent two major topics of Large Helical Device (LHD) towards fusion relevant conditions, high-density operation and high-ion-temperature operation, are reported. Super dense core plasma was obtained by the combination of repetitive hydrogen ice pellet injection and high power neutral beam injection (NBI) heating. A very peaked density profile with the highest central density of $1.1 \times 10^{21} \mathrm{~m}^{-3}$ was produced showing that the particle transport was suppressed very well in the plasma core. The spatial density profile varies as the position of magnetic axis $\left(R_{\mathrm{ax}}\right)$, and the steepest profile is obtained at $R_{\mathrm{ax}}=3.95 \mathrm{~m}$. The highest central ion temperature of $5.6 \mathrm{keV}$ was obtained in hydrogen plasma at electron density of $1.6 \times 10^{19} \mathrm{~m}^{-3}$ by NBI, where a peaked iontemperature profile with internal ion energy transport barrier was observed. The profile of electron temperature did not change much and was broad even when the ion temperature had a peaked profile. The central ion temperature is higher than the electron temperature, which is a new operation regime of LHD. High central ion temperature accompanied strong toroidal rotation and an extreme hollow profile of carbon ions (impurity hole). These steep temperature profiles were obtained so far at around $R_{\mathrm{ax}}=3.6 \mathrm{~m}$. The compatibility between particle and energy confinement is a new issue of LHD to explore a new operation regime for attractive fusion reactor.
\end{abstract}

(C) 2009 The Japan Society of Plasma Science and Nuclear Fusion Research

Keywords: helical system, density limit, internal diffusion barrier, internal transport barrier, plasma rotation, impurity hole

DOI: $10.1585 /$ pfr.4.027

\section{Introduction}

The Large Helical Device (LHD) experiment started in 1998 to address the fundamental physical issues to re-

author's e-mail: Kaneko.osamu@nifs.ac.jp

*) This article is based on the invited talk at the 14th International Congress on Plasma Physics (ICPP 2008). alize magnetically confined toroidal fusion reactor, i.e., steady state operation, high- $\beta$ plasma confinement, and control of power fluxes to walls [1]. These activities are carried out in different manner from tokamaks because LHD deals with net-current free plasma [2]. Now LHD is the only device that can produce fusion relevant plasma 
other than tokamak.

Nuclear fusion enters a new phase of research handling burning plasma. The ITER project has started on the basis of many experimental data from present tokamaks. However, some issues are still remained unsolved and more research will be needed. It is expected that the results of LHD contribute to comprehensive understanding of magnetically confined toroidal fusion plasmas, and bring about solutions of present issues.

LHD is the world's largest superconducting magnetic confinement device, which employs a heliotron configuration, the major and minor radii of which are $3.75 \mathrm{~m}$ and $0.64 \mathrm{~m}$, respectively. A confining magnetic field of up to $3 \mathrm{~T}$ is provided steadily by means of the fully superconducting coil system. The current heating capability is $21 \mathrm{MW}$ of NBI, $3 \mathrm{MW}$ of ICRF and $2 \mathrm{MW}$ of ECH. NBI consists of four beam lines, three of which are high energy tangential beam lines using negative ion technology, and one of which is a low energy radial beam line with conventional positive ion technology. ICRF consists of three pairs of half-turn antenna, and ECH consists of $84 \mathrm{GHz}, 87 \mathrm{GHz}$ and $168 \mathrm{GHz}$ gyrotrons. Both ICRF and ECH are capable of steady state operation [3]. LHD is equipped with two different divertor systems; the Helical Divertor (HD) and the Local Island Divertor (LID) [4]. The HD has a shape similar to the double-null open divertor in tokamaks except for its helically twisted structure. The LID uses an $m / n=1 / 1$ magnetic island produced by the externally applied resonant field to guide particle and heat fluxes to the locally inserted divertor plates.

Through the decade of experiment, LHD has demonstrated a remarkable progress in the issues addressed. A very long pulse operation by means of ICRF close to one hour with a heating power of $500 \mathrm{~kW}$ was achieved, where the investigation of highly energetic particles and control of the heat load on the divertor plates have made a significant contribution [5]. High $\beta$ discharges also have been advanced by exploration of aspect-ratio control [6] and consequently a beta of $5.0 \%$ has been achieved. The understanding of pressure driven MHD modes has been deepened through the research in the high $\beta$ regime.

These results were obtained through optimization of confining magnetic field structure of LHD. The most important control parameter is the position of magnetic axis $\left(R_{\mathrm{ax}}\right)$. It is known that the inward shift of $R_{\mathrm{ax}}$ reduces the excursion of particle orbit from magnetic flux surface although the magnetic well disappears. The experimental results show that the global energy confinement also depends on $R_{\mathrm{ax}}$, and is improved when $R_{\mathrm{ax}}$ is shifted inward. One of the most important findings in LHD is that the plasma is still stable at small $R_{\mathrm{ax}}$ although Mercier criterion indicates unstable there [7]. Because the inward shift favors plasma heating from the view point of confining high energy ions, the optimization of both heating and confinement comes out successfully at the same time [8].

\section{Extension of Improved Confine- ment Regime}

Efforts in extending improved confinement regime have been made on increasing plasma density and ion temperature, recently. The optimization of $R_{\mathrm{ax}}$ has been carried out as before, and the maximum ion temperature was obtained at around the position of maximum energy confinement. On the other hand, the behavior of particle confinement looks different from that of energy confinement. The highest density was obtained when $R_{\mathrm{ax}}$ is shifted outward. These results are summarized in Fig. 1.

One of the highlighted topics in recent experiments is the discovery of a Super-Dense-Core (SDC) plasma operation created by an Internal Diffusion Barrier (IDB) $[9,10]$. Combination of highly efficient wall pumping and core fueling by repetitive ice pellet injection generates the IDB [11] with a central density of more than $1 \times 10^{21} \mathrm{~m}^{-3}$. A steep gradient in the density profile is formed showing a drastic improvement in particle transport. It clearly demonstrates that helical plasma has an advantage of high density operation, and the design of an attractive reactor operating with high density and relatively low temperature becomes possible. Simultaneously, the IDB is attracting keen interest into the physics of its transition and structural formation. Through the campaign of research such high density achievement, the highest fusion triple product was also obtained.

The other recent topic is the improvement of ion energy confinement. To sustain plasma of high ion temperature is also an important issue of LHD as a fusion plasma confining device. Since main heating power of LHD is supplied by $180-\mathrm{keV}$ hydrogen NBI, most of the power is absorbed by electrons. Therefore we had carried out ion heating using Ar target plasma (it is possible in LHD) to increase the direct ion heating power, and demonstrated that the highest central ion temperature of $13.5 \mathrm{keV}$ was obtained at the electron density of $0.3 \times 10^{19} \mathrm{~m}^{-3}$ in 2004 . Recently, a new beam line of $40 \mathrm{keV}$ hydrogen has been installed for ion heating as well as charge exchange recombi-

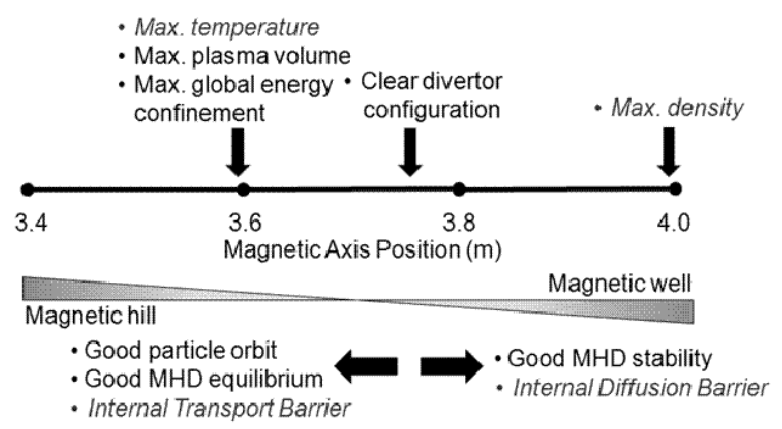

Fig. 1 Summary of dependence of performance of LHD plasma on magnetic axis position. Recent topics are shown in Italic. 
Table 1 Achieved plasma parameters in LHD.

\begin{tabular}{|l|c|c|}
\hline & Achieved & Goal \\
\hline $\begin{array}{l}\text { Central ion } \\
\text { temperature }\end{array}$ & $\begin{array}{c}5.6 \mathrm{keV} \\
\left(1.6 \times 10^{19} \mathrm{~m}^{-3}\right) \\
13.5 \mathrm{keV}[\mathrm{Ar}] \\
\left(3 \times 10^{18} \mathrm{~m}^{-3}\right)\end{array}$ & $\begin{array}{c}10 \mathrm{keV} \\
\left(2 \times 10^{19} \mathrm{~m}^{-3}\right)\end{array}$ \\
\hline $\begin{array}{l}\text { Central electron } \\
\text { temperature }\end{array}$ & $\begin{array}{c}10 \mathrm{keV}^{18} \\
\left(5 \times 10^{18} \mathrm{~m}^{-3}\right)\end{array}$ & $\begin{array}{c}10 \mathrm{keV} \\
\left(2 \times 10^{19} \mathrm{~m}^{-3}\right)\end{array}$ \\
\hline $\begin{array}{l}\text { Central electron } \\
\text { density }\end{array}$ & $\begin{array}{c}1.1 \times 10^{21} \mathrm{~m}^{-3} \\
\left(T_{\mathrm{e}}=0.3 \mathrm{keV}\right) \\
1 \times 10^{20} \mathrm{~m}^{-3} \\
\left(T_{\mathrm{e}}=1.5 \mathrm{keV}\right)\end{array}$ & $\begin{array}{l}1 \times 10^{20} \mathrm{~m}^{-3} \\
\left(T_{\mathrm{e}}=1 \mathrm{keV}\right)\end{array}$ \\
\hline Volume averaged $\beta$ & $5.0 \% 0.425 \mathrm{~T}$ & $>5 \% 1-2 \mathrm{~T}$ \\
\hline $\begin{array}{l}\text { Long pulse } \\
\text { operation }\end{array}$ & $54 \mathrm{~min} .28 \mathrm{sec}$. & $1 \mathrm{hour}$ \\
\hline Stored energy & $(490 \mathrm{~kW})$ & $(3,000 \mathrm{~kW})$ \\
\hline Plasma pressure & $1.6 \mathrm{MJ}$ & \\
\hline $\begin{array}{l}\text { Fusion } \\
\text { triple product }\end{array}$ & $5 \times 10^{19} \mathrm{keVa}$ & {$\left[n(0) \tau_{\mathrm{E}} T(0)\right]$} \\
\hline
\end{tabular}

nation spectroscopy to measure the ion temperature profile. Then the campaign of high $T_{\mathrm{i}}$ achievement has been revisited by using hydrogen plasma. As a result, highest central ion temperature of $5.6 \mathrm{keV}$ at the moderate electron density of $1.6 \times 10^{19} \mathrm{~m}^{-3}$ was obtained. It was found that the high ion temperature accompanies an ion energy transport barrier. This improvement does not seem to affect electron transport, but interestingly induces out flux of carbon ions.

These two topics will be described in detail in later sections. The latest achievement in LHD is summarized in Table 1 . They are obtained by increasing heating power, development of diagnostics, and improving operational scenario including fueling technology.

\section{Internal Diffusion Barrier - Super Dense Core plasma -}

The internal Diffusion Barrier appears in NBI heated plasmas in LHD as a peaked, high density profile, which is generated by multiple pellet injections from the outside mid-plane under good wall condition. At first IDB was found when powerful particle control was done by using the Local Island Divertor (LID) [12]. Later, it was found also without LID, that is, in the configuration of intrinsic helical divertor when the wall is conditioned well. Typical waveforms are illustrated in Fig. 2. In this case, seven pellets are injected sequentially, and density increases stepwise as each pellet shot. The central electron density has reached its maximum just after the termination of a sequence of pellet injections, and then the central density decays slowly with a time constant of $1 \mathrm{~s}$. On the other hand, the electron and ion temperature increase after the termination of a sequence of pellet injections, and the plasma stored energy reaches its maximum about $0.1 \mathrm{~s}$ later from

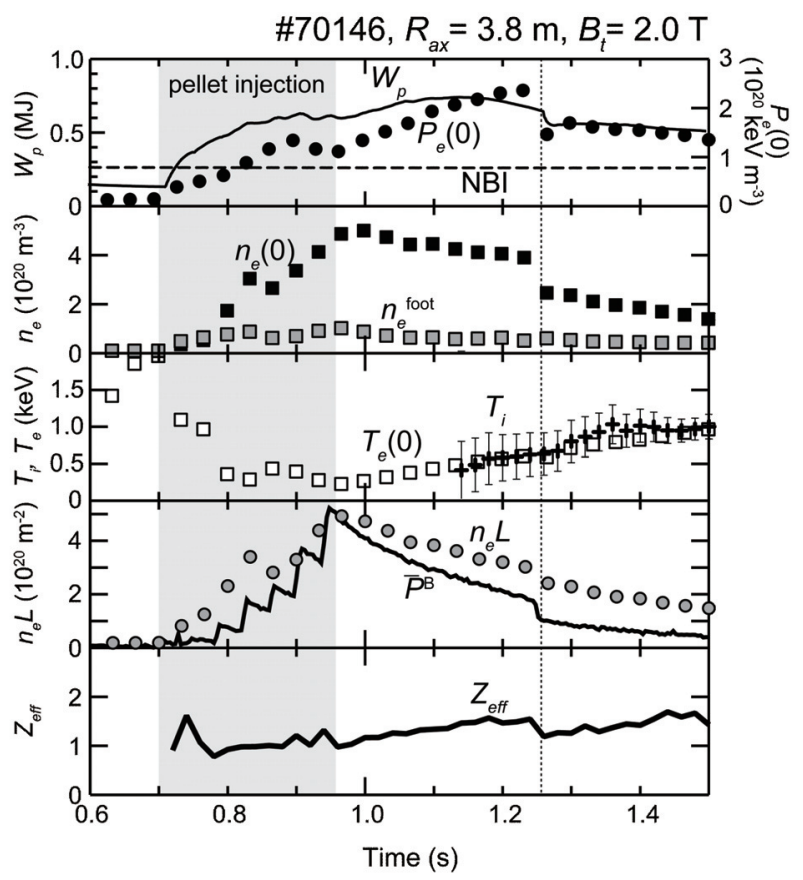

Fig. 2 Typical time behavior of plasma parameters of discharge with IDB. The central density takes its maximum just after the termination of sequential pellet injection, while the central pressure in the density decay phase.

the time of termination.

The obtained density and temperature profiles are shown in Fig. 3 compared with those of without IDB. The clear jump in the density gradient is observed in IDB discharge. In contrast to the density profile, electron temperature profile does not depend much on the presence of IDB. In the optimized discharge, the maximum central density is reached $1.1 \times 10^{21} \mathrm{~m}^{-3}$. Despite of such an extremely high density, the central electron temperature remains $0.3 \mathrm{keV}$, and as a result the central plasma pressure reaches $150 \mathrm{kPa}$.

The plasmas with the IDB also exhibit high fusion plasma performance. In the density decay phase after pellet injection, good energy confinement recovers when the heating power is reduced and the highest central fusion triple product of $n(0) \tau_{\mathrm{E}} T(0)=5 \times 10^{19} \mathrm{keVm}^{-3} \mathrm{~s}$ is obtained.

It is interesting to compare these profiles of SDC plasma with other high performance plasma. Figure 4 shows density and electron temperature profile of plasma with maximum stored energy in LHD (1.6 MJ). There is no jump in density and temperature gradients, and both profiles have a bell shape. It should be noted that the stored energy of SDC plasma becomes small since effective plasma volume is narrow as can be seen in Fig. 5 .

The optimization of this multi-pellet injection scenario has been carried out on the magnetic axis position, and it was found that clear IDB is generated when the magnetic axis is shifted outward in spite of reduction of global energy confinement. Figure 5 shows the change of 


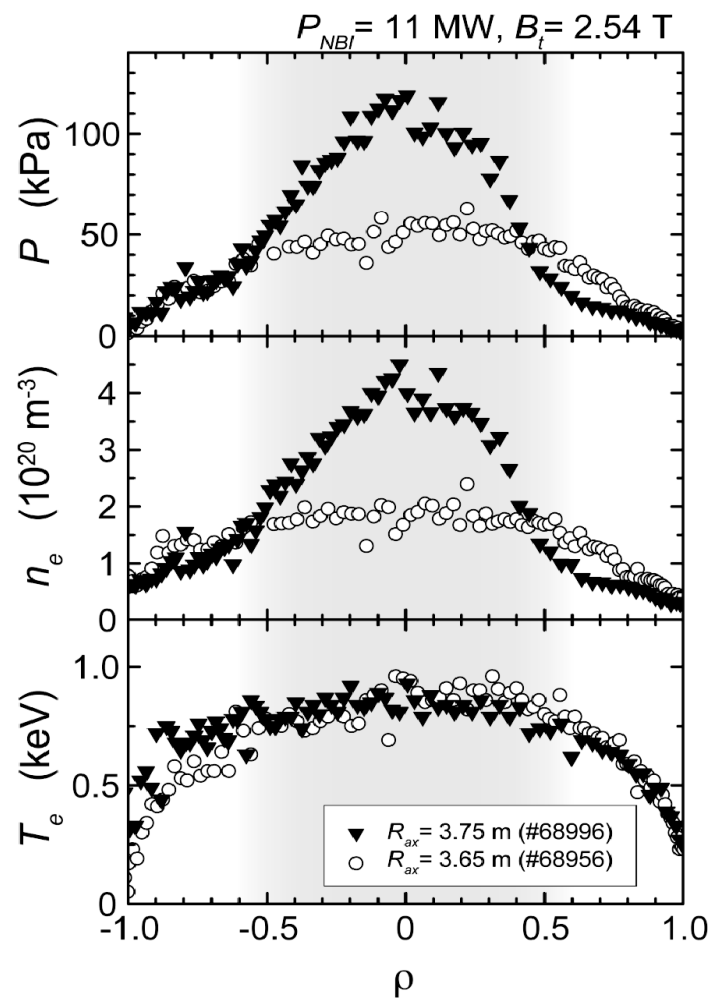

Fig. 3 The density and temperature profiles in SDC plasma $\left(R_{\mathrm{ax}}=3.75 \mathrm{~m}\right)$ are compared with those of standard discharge $\left(R_{\mathrm{ax}}=3.6 \mathrm{~m}\right)$. Shaded is the region where IDB exists in the case $R_{\mathrm{ax}}=3.75 \mathrm{~m}$

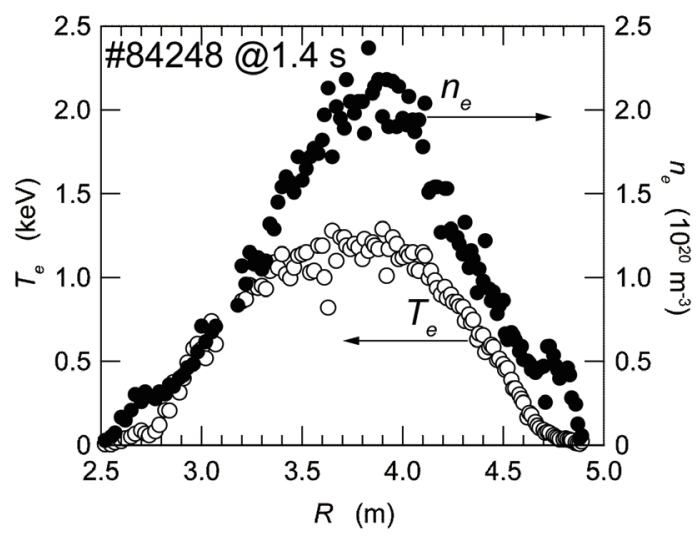

Fig. 4 Typical density and temperature profiles of plasma having large stored energy.

electron density profile as the position of magnetic axis varies. When $R_{\mathrm{ax}}$ shifts outward from the standard position of $3.6 \mathrm{~m}$, IDB appears locally in the plasma at first, and the area of IDB expands and central density increases as $R_{\mathrm{ax}}$. The obtained density profile becomes very steep and large Shafranov shift is observed. One of the specific features of IDB is that it has a clear location of changing density gradient $\left(R_{\text {foot }}\right)$. This position increases with $R_{\mathrm{ax}}$ as can be seen in Fig. 5. $R_{\text {foot }}$ does not correspond to the ab-

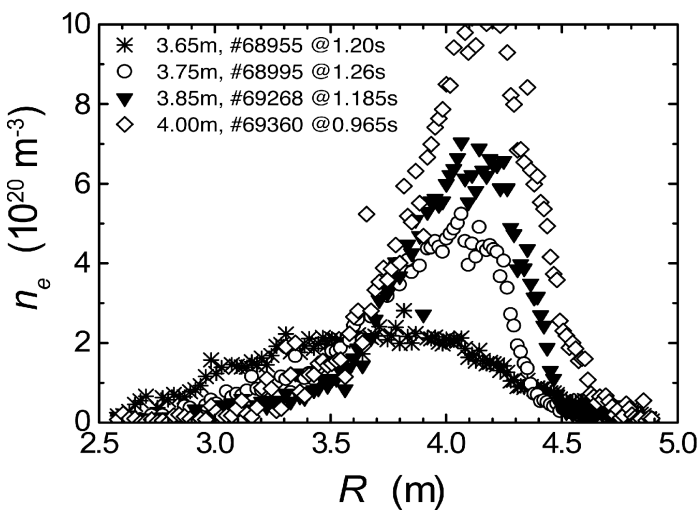

Fig. 5 Dependence of density profile on magnetic axis position. Conditions of injecting NB power and pellet fuelling are almost the same. A large Shafranov shift is observed for SDC plasma.

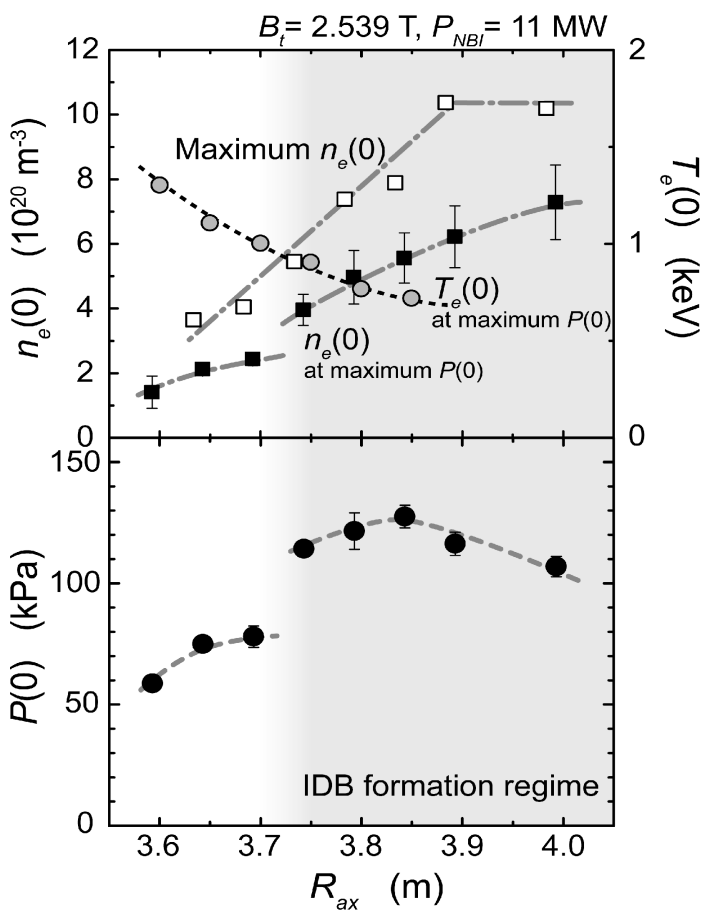

Fig. 6 Dependence of central plasma parameters on magnetic axis position. Shaded is the region where the IDB is observed.

solute values of local density or electron temperature. At $R_{\text {ax }}=4.0 \mathrm{~m}, R_{\text {foot }}$ is almost located at the last closed flux surface.

The dependence of central electron density and temperature on $R_{\mathrm{ax}}$ is shown in Fig. 6. IDB can be observed when $R_{\mathrm{ax}}$ is larger than $3.75 \mathrm{~m}$. As described before, the maximum central pressure is obtained during the decay of electron density after sequential pellet injection, and it is optimal at $R_{\mathrm{ax}}=3.85 \mathrm{~m}$. It is noted again that the global energy confinement becomes worse when $R_{\mathrm{ax}}$ increases. It is observed in LHD that the favorable density dependence 
on energy confinement ( $\tau_{\mathrm{E}} \sim n_{\mathrm{e}}^{0.6}$ ) disappears at high density in gas puff discharges, and pellet fueling recovers this dependence. However, pellet fueling does not increases an improvement factor of ISS95 further. It is also the case of SDC discharges even in the presence of IDB.

One of the reasons why very high central density is obtained in outer shift plasma is that the center fueling is effectively performed. In SDC plasma, electron temperature is lower than $0.5 \mathrm{keV}$ at which pellets can reach the center of plasma. The main heating source is tangential $\mathrm{NB}$, and its tangency radius is $3.65-3.7 \mathrm{~m}$. Therefore the heating profile becomes peaked when the magnetic axis is shifted outward. Although beam deposition profile becomes flat or even hollow when the electron density becomes high, very steep density profile mitigates this surface absorption. These two conditions being combined, favorable central particle fueling is realized and this may result in high central density. In fact it was found experimentally that central density does not become high when the central electron temperature is lower than $0.3 \mathrm{keV}$. This is because the electron temperature is too low for the pellet to be ablated in the plasma center.

The SDC plasma is unique and different from conventional helical plasma. The empirical density limit in helical systems based on the experimental results in medium size helical systems was proposed by Sudo [13]. This scaling, defined by $n_{\mathrm{e}, \text { Sudo }}\left[10^{20} \mathrm{~m}^{-3}\right]=0.25\left(P_{\mathrm{abs}}[\mathrm{MW}] B[\mathrm{~T}] /\right.$ $\left.\left(a[\mathrm{~m}]^{2} R[\mathrm{~m}]\right)\right)^{0.5}$, is considered to reflect the situation that the operational density is limited by the power balance between the heating power and the radiation loss at the plasma periphery. The obtained averaged density of SDC plasma is much higher than Sudo limit, and this is because the electron density at the periphery is small enough to suppress the radiation loss, which is typically $2-4 \times 10^{19} \mathrm{~m}^{-3}$ even if the central density is $1 \times 10^{21} \mathrm{~m}^{-3}$. It is experimentally shown that the density collapse or detachment occurs when the density of plasma edge $\left(T_{\mathrm{e}} \sim 100 \mathrm{eV}\right)$ exceeds a local Sudo scaling [14].

A simple theoretical analysis on SDC plasma has been done [15]. It is noted that IDB plasma has high collisionality $\left(v_{\mathrm{b}}<10\right)$. A pulsed local particle source in the center of plasma may generate a peaked profile in density and a hollow profile in temperature instantaneously. In such a case large positive electric field at the center and strong electric field shear are predicted from the neoclassical (NC) ambipolar condition. This electric field shear may reduce the anomalous diffusion coefficient and can keep the steep gradient of density. Experimentally, it is very difficult to measure radial electric field in such high density plasma, and we do not have evidence yet.

\section{Improvement of Ion Energy Trans- port - High Ion Temperature -}

LHD plasmas typically have higher electron temperature than ion temperature because the electron heating is dominant by the high-energy neutral beam injection (180 keV, hydrogen). The NC ambipolar radial electric field $\left(E_{\mathrm{r}}\right)$ in such $T_{\mathrm{e}}>T_{\mathrm{i}}$ plasmas tends to be positive (electron root), and the impact of the positive $E_{\mathrm{r}}$ on confinement properties and improvement have been extensively studied in LHD [16-19].

On the other hand, helical plasmas with $T_{\mathrm{i}}>T_{\mathrm{e}}$ are predicted to have a negative ambipolar $E_{\mathrm{r}}$. In this regard, the ion heating experiments in LHD are important not only to demonstrate the high- $T_{\mathrm{i}}$ plasma confinement capability, but also to extend the plasma parameter regime to $T_{\mathrm{i}}>T_{\mathrm{e}}$ so that the impact of $E_{\mathrm{r}}$ on helical plasma confinement can be widely studied.

To demonstrate high- $T_{\mathrm{i}}$ plasma confinement capability in LHD, high- $Z$ such as $\mathrm{Ne}$ and Ar plasmas with low ion density (in the range of $10^{18} \mathrm{~m}^{-3}$ ) were used as targets during the period when only high-energy tangential NBIs were operating, to increase the absorption power per ion effectively. The central $T_{\mathrm{i}}$ of about $13.5 \mathrm{keV}$ was successfully demonstrated by this approach [20].

Through the study of high- $Z$ plasma ion heating, it was found that the central ion temperature is proportional to the absorbed power per ion regardless of the ion species, which is different from the empirical scaling for energy confinement ISS95. This successful proof-of-principle ion heating experiment has encouraged the installation of lowenergy $40-\mathrm{keV}$ perpendicular NBI to increase the direct ion-heating power. As a result, $T_{\mathrm{i}}$ has now exceeded $5 \mathrm{keV}$ in hydrogen plasma, which extends the high- $T_{\mathrm{i}}$ regime in LHD to higher density region. Also, the core of the high$T_{\mathrm{i}}$ plasma is characterized by $T_{\mathrm{i}} / T_{\mathrm{e}}>1$, which provides a new parameter regime for LHD plasmas. It should also be noted that this low-energy NBI is utilized for the charge exchange recombination spectroscopy (CXS) for measuring radial profiles of $T_{\mathrm{i}}$, toroidal rotation $\left(V_{\mathrm{t}}\right)$ and poloidal rotation to evaluate radial electric field. The observation of $T_{\mathrm{i}}$ and $V_{\mathrm{t}}$ profiles has enhanced the physics understandings of ion dynamics in helical plasmas.

Figure 7 shows an example of operational scenario of high- $T_{\mathrm{i}}$ discharge. For this discharge, the carbon pellet is injected at $1.8 \mathrm{~s}$ to the target hydrogen plasma sustained with 2 NBs. During the density decay phase, one additional tangential NBI and perpendicular NBI are superposed to heat ions as shown in Fig. 7. The perpendicular NB is modulated to obtain the background signals of CXS measurement. The electron temperature in the core region is almost unchanged (3-4 keV) even during the phase of $T_{\mathrm{i}}$ increase, to result in the condition of $T_{\mathrm{i}} / T_{\mathrm{e}}>1$. At this high- $T_{\mathrm{i}}$ phase, carbon density is significantly reduced as described later. It can be seen from the figure that the ion temperature at periphery does not change, which means the temperature gradient becomes steeper. The high- $T_{\mathrm{i}}$ state with $\sim 5 \mathrm{keV}$ is kept for $\sim 100 \mathrm{~ms}$ as shown in Fig. 7 .

Typical spatial profiles of $T_{\mathrm{i}}, T_{\mathrm{e}}$ and $n_{\mathrm{e}}$ at the time of maximum $T_{\mathrm{i}}$ are illustrated in Fig. 8. The spatial profile of ion temperature varies in the time scale of energy confine- 


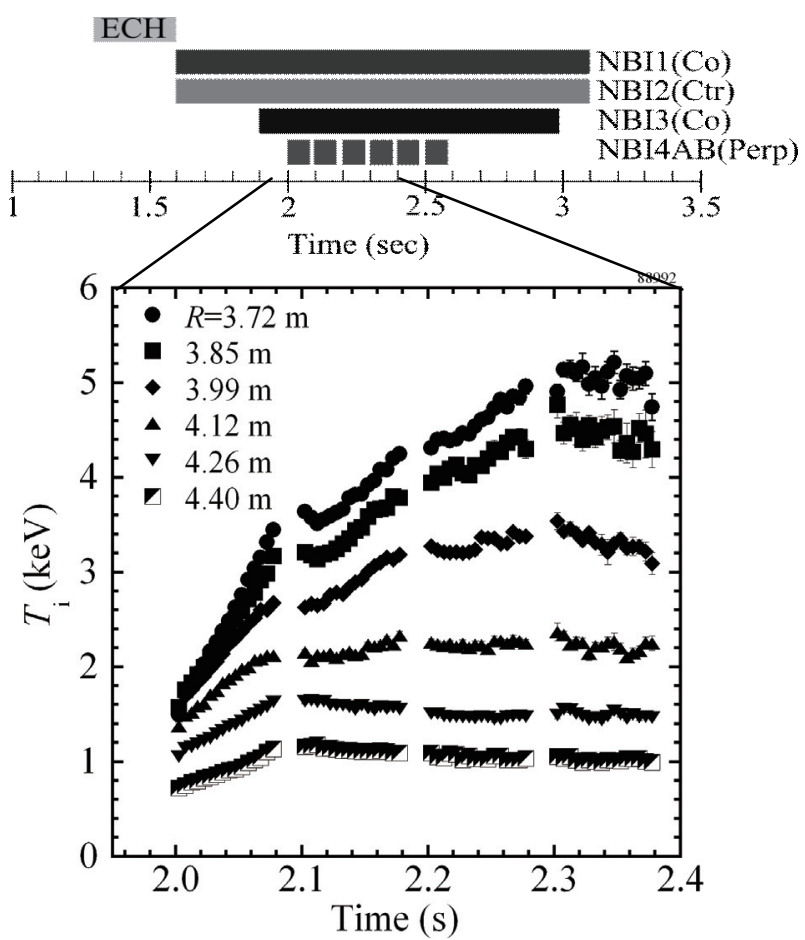

Fig. 7 Operation scenario of high $T_{\mathrm{i}}$ plasma production. Combination of four NBs is illustrated. $R_{\mathrm{ax}}=3.60 \mathrm{~m}$ and $B_{\mathrm{T}}=-2.75 \mathrm{~T}$.

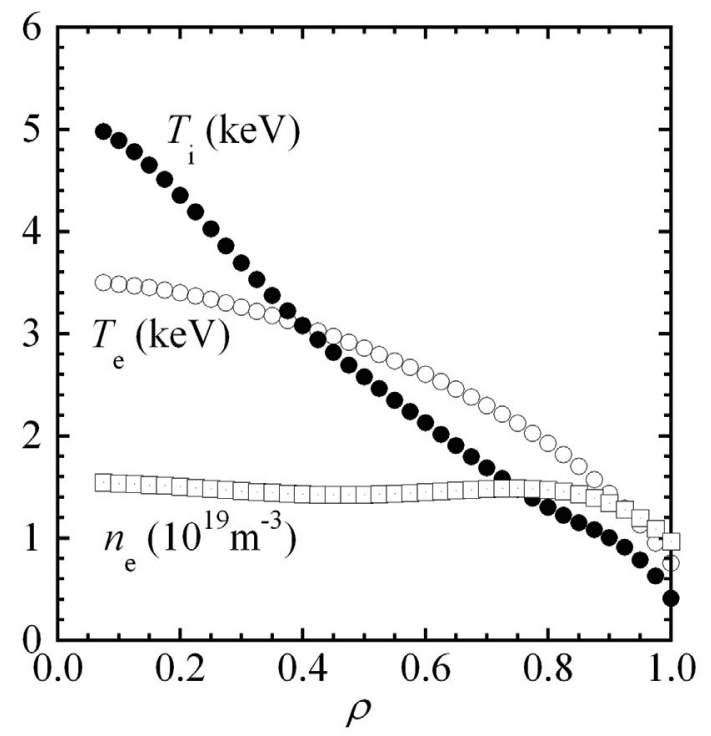

Fig. 8 Typical $T_{\mathrm{e}}, T_{\mathrm{i}}$, and $n_{\mathrm{e}}$ profiles of a high- $T_{\mathrm{i}}$ discharge.

ment time.

The density scan experiments were also performed and the results are summarized in Fig. 9, where $T_{\mathrm{i}}$ and $T_{\mathrm{e}}$ in the core region are plotted as a function of the lineaveraged electron density, $\bar{n}_{\mathrm{e}}$. In this range of electron density, central electron temperature lay between $3 \mathrm{keV}$ and $4 \mathrm{keV}$ and the dependence on density is rather weak. It is clearly seen that plasmas with $T_{\mathrm{i}}>T_{\mathrm{e}}$ are generated at

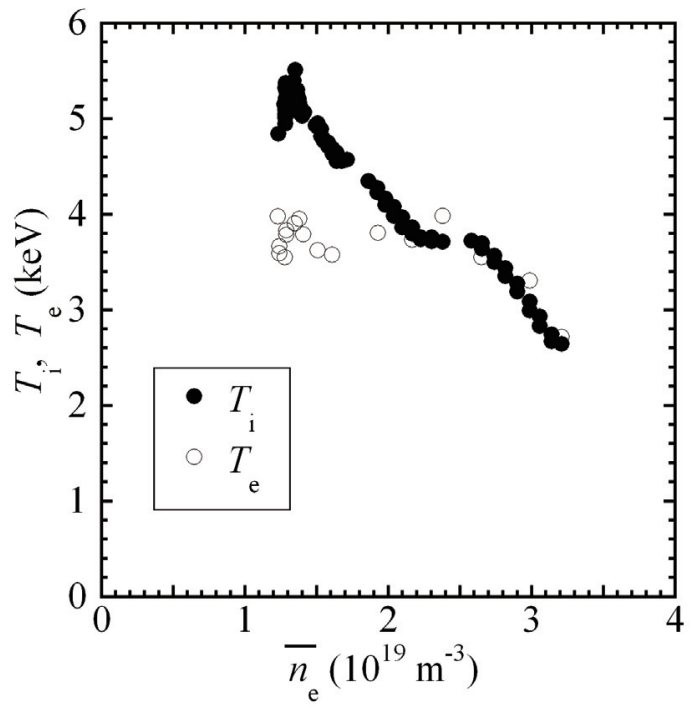

Fig. 9 Dependence of measured $T_{\mathrm{i}}$ and $T_{\mathrm{e}}$ at the core region on the line-averaged electron density in high- $T_{\mathrm{i}}$ discharges.

$\bar{n}_{\mathrm{e}}<2 \times 10^{19} \mathrm{~m}^{-3}$. As the density increases, $T_{\mathrm{i}}$ becomes comparable to $T_{\mathrm{e}}$ due to the energy transfer between electrons and ions.

It is recognized that the temperature gradient changes at $\rho \sim 0.7$ in Fig. 8, indicating that the ion heat confinement property changes qualitatively by this "foot point." By evaluating the $\chi_{i_{-} \text {exp }}$ with the locally measured $T_{\mathrm{i}}$ gradient, the spatial jump and the change of the slope of $\chi_{i_{-} \text {exp }}$ are recognized. Based on these facts, it can be concluded that high- $T_{\mathrm{i}}$ plasma is obtained as a result of the confinement improvement, not just a result of the increase of heating power.

It is important to investigate the contribution from the $\mathrm{NC}$ transport in helical plasmas especially in the low collisionality regime since the enhancement of heat and particle diffusion is anticipated due to the predicted ripple transport with an unfavorable dependence on $1 / v$, where $v$ is the collision frequency. The experimentally evaluated ion thermal diffusivity $\chi_{\mathrm{i}}$ is compared with NC calculation in Fig. 10. For the experimental ion thermal diffusivity $\left(\chi_{\text {i_exp }}\right)$ analysis, an NBI deposition calculation is performed using the FIT code [21], which can analyze the NBI heating profiles in a 3D magnetic configuration assuming quasi-steady-state plasma. Here, $Z_{\text {eff }}$ of the background plasma is assumed to be 2 . It has been confirmed that changing $Z_{\text {eff }}$ from 2 to 3 gives only a slight change in the deposited power and therefore the estimation of $\chi_{\text {i_exp }}$.

The NC transport analysis has been performed using the GSRAKE code [22]. The NC diffusion coefficients are evaluated by utilizing the experimentally obtained density and temperature profiles to obtain the electron and ion particle fluxes $\Gamma_{\mathrm{e}}$ and $\Gamma_{\mathrm{i}}$. The ambipolar condition, $\Gamma_{\mathrm{e}}=\Gamma_{\mathrm{i}}$, provides the ambipolar $E_{\mathrm{r}}$. It is fair to think that the $\mathrm{NC}$ ambipolar $E_{\mathrm{r}}$ gives a rather good indication for the experi- 

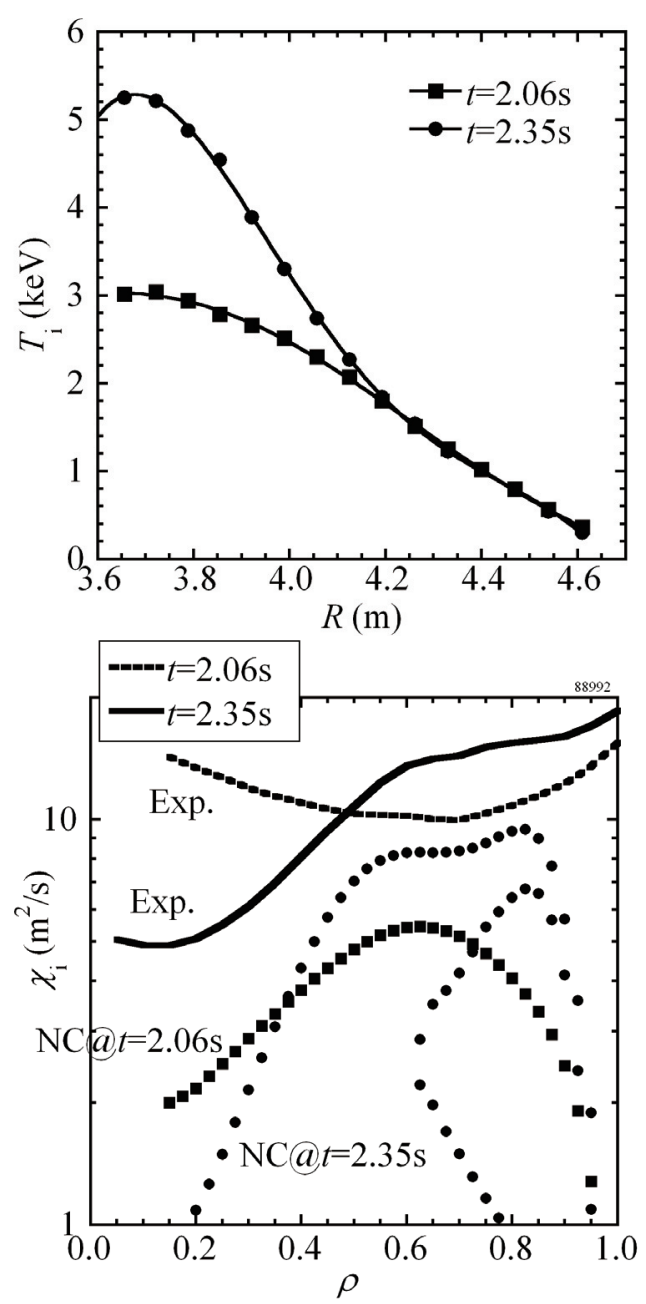

Fig. 10 Ion thermal diffusivity before and after ITB formation are compared with $\mathrm{NC}$ evaluation. Upper figure shows experimentally observed $T_{\mathrm{i}}$ profiles.

mentally observed $E_{\mathrm{r}}$ in LHD [23]. It is also noted that, recently, negative $E_{\mathrm{r}}$ is evaluated in the core region from the potential profile measured by the Heavy Ion Beam Probe (HIBP).

The NC ion thermal diffusivity $\left(\chi_{\mathrm{i}_{-} \mathrm{NC}}\right)$ shows little change in the core region between the two cases in Fig. 10. It should be also noted that $\chi_{\mathrm{i}_{\mathrm{N}} \mathrm{NC}}$ is predicted to be larger in the higher- $T_{\mathrm{i}}$ case if $E_{\mathrm{r}}$ is assumed to be zero due to the temperature scaling of $1 / v$ ripple transport. The ambipolar $E_{\mathrm{r}}$ plays a significant role in preventing the theoretically predicted "pure" ripple transport. It is predicted theoretically that $\chi_{\mathrm{i}_{N} \mathrm{NC}}$ can be reduced when the $T_{\mathrm{i}} / T_{\mathrm{e}}$ increases. Therefore, higher $T_{\mathrm{i}}$ is still possible when the ion heating power increases. Experimentally, ion heating is not very efficient when $T_{\mathrm{i}} / T_{\mathrm{e}}$ is small $(<1)$.

The toroidal plasma rotation velocity $V_{\mathrm{t}}$ is also largely enhanced in the plasma core in the direction corresponding to the predominance of tangentially injected neutral beams (co or counter direction). The observed $V_{\mathrm{t}}$ becomes large
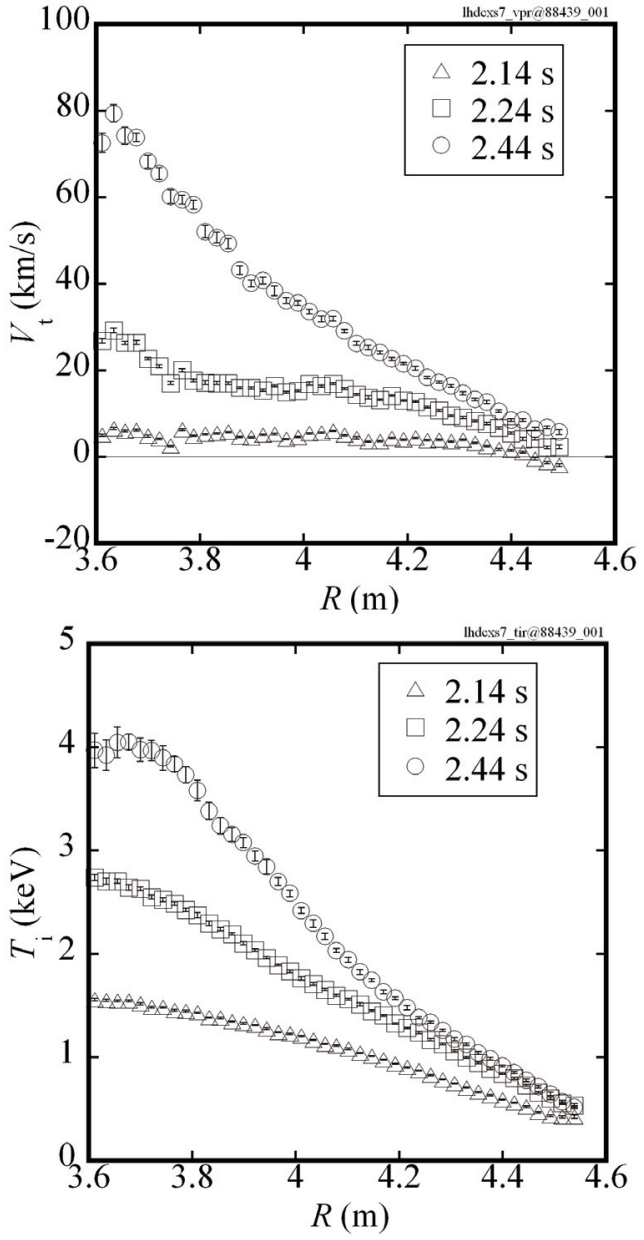

Fig. 11 Spatial profile of toroidal rotation and ion temperature at corresponding times. As $T_{\mathrm{i}}$ increases, $V_{\mathrm{t}}$ also increases although the heating condition is the same.

as the ion temperature increased although the condition of injected NB is almost the same. Also the spatial profile of $V_{\mathrm{t}}$ is similar to that of ion temperature, which is shown in Fig. 11. This suggests that the toroidal viscosity and radial momentum transport are improved in the same way as ion energy diffusivity.

The spontaneous rotation is of great interest in the area of momentum transport physics in toroidal system. In LHD, three types of toroidal flows were observed. In the core region, the toroidal flow is driven by the external momentum input of tangential NBI. On the other hand, at plasma periphery, the toroidal flow driven by radial electric field was observed, where the plasma flows along the line that the variation of magnetic field strength is minimum. In LHD it is co direction when the negative electric field is produced. Recently, the other spontaneous toroidal flow was observed in the middle of plasma, which is driven by the ion temperature gradient [24]. This spontaneous flow is induced in co-direction as the absolute value of ion temperature gradient increases. This tendency does not depend 
on magnetic axis position.

The improved high- $T_{\mathrm{i}}$ discharge also reveals a specific phenomenon that spatial profile of carbon ions shows an extreme hollow profile (we call it "impurity hole"). Figure 12 shows a change of measured carbon ion profiles before and after ITB formation in two cases of different magnetic axis. It is clearly seen that extreme hollow profile appears with ITB even though electron density profile is almost flat. The carbon component reduces less than $1 \%$ in the center of plasma. From these observations the profile of hydrogen ion is deduced to be a weakly peaked profile. It is noted that the time scale of forming such hollow profile is the same as that of peaking of ion temperature. A simple analysis shows that the diffusion coefficient of carbon reduces from $1 \mathrm{~m}^{2} / \mathrm{s}$ at the plasma edge to $0.1 \mathrm{~m}^{2} / \mathrm{s}$ in the core of plasma while there exists an outer flow of several $\mathrm{m} / \mathrm{s}$ throughout this region.

In the figure, it is noted that the impurity hole is deeper at $R_{\mathrm{ax}}=3.575 \mathrm{~m}$ than $3.55 \mathrm{~m}$. This is different trend from hydrogen main plasma, which tends to have hollow density profile when the magnetic axis is shifted inward [25].

This "impurity hole" may also exist in $\mathrm{Ne}$ and $\mathrm{Ar}$ plasma. In LHD, central ion temperature is also measured by using Doppler broadening of Ar XVII. In high- $T_{\mathrm{i}}$ experiment with Ar target plasma, we observed a transient
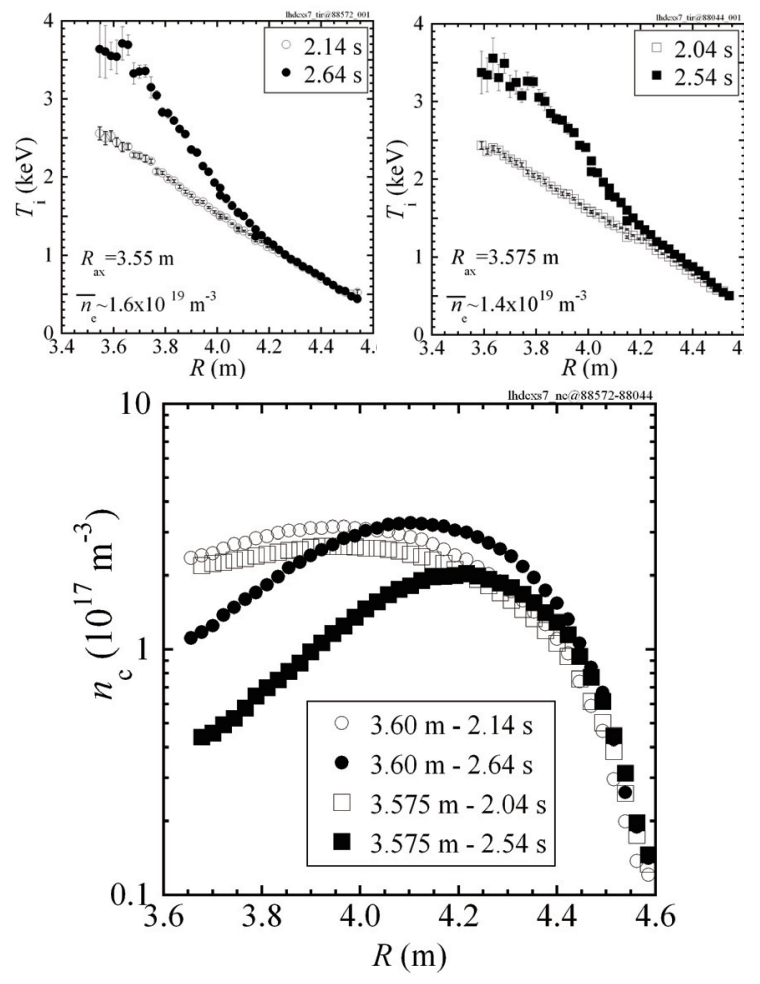

Fig. 12 Formation of impurity hole at high ion temperature. Comparison is made for two $R_{\mathrm{ax}}$ cases $(3.55 \mathrm{~m}$ and $3.575 \mathrm{~m}$ ), for which $T_{\mathrm{i}}$ profiles are shown in the upper figures. Symbols in lower figures correspond to those of upper figures. behavior of achieving very high ion temperature following abrupt decay, which is probably due to the impurity hole and the observation point may be no more the plasma center. The NC particle transport theory of helical system permits the case of outward flow of heavy ions in light ions [26]. This is different from tokamaks where heavy ions tend to accumulate in the center. However the obtained profile is too hollow to be explained. Additional convection mechanism is needed.

\section{Conclusions - Compatibility be- tween Particle and Energy Confine- ment -}

The LHD has succeeded to expand its operational regimes in high-density operation and high-iontemperature operation. In both regimes improvement of transport is observed. An Internal Diffusion Barrier by combination of efficient wall pumping and core fueling by pellet injection has realized a super dense core as high as $1.1 \times 10^{21} \mathrm{~m}^{-3}$, which explores a new concept of "super dense core helical reactor" with a density of the order of $1 \times 10^{21} \mathrm{~m}^{-3}$ and a temperature of less than $10 \mathrm{keV}$. Such operation has an advantage that high density mitigates plasma wall interaction (reduction of erosion, etc.), and reduces engineering demand of a reactor. An Internal Ion Transport Barrier by strong ion heating has revealed a large outflow of carbon ions in plasma forming "impurity hole."

The optimization of these operational conditions was carried out through the change of magnetic axis position which is one of the most important control parameters in LHD. It is well known that the position of magnetic axis affects MHD characteristics as well as particle orbit

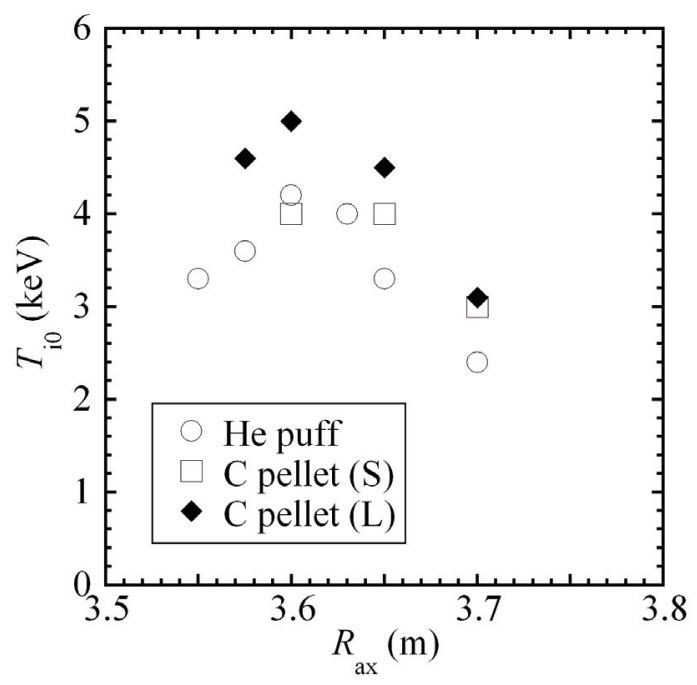

Fig. 13 Dependence of $T_{\mathrm{i}}(0)$ on magnetic axis position, where different symbols correspond to different experimental scenarios examined. 
in LHD [8]. Since the dependence of these two important characteristics on magnetic axis is reciprocal, the compatibility between two was the big issue. Fortunately, it was experimentally observed that the MHD constraint is not critical, and that the condition of optimal global energy confinement coincides with that of optimal particle orbit. The issue of compatibility was solved by inward shift of magnetic axis.

However, recent two experimental results face us a new issue. The IDB reveals in outer-shifted plasma while the ion ITB appear in inner-shifted plasma (Fig. 13). The elucidation of this compatibility would be a key of further progress of LHD towards fusion reactor. The experimental results suggest that the central heating and fueling is the key point to realize IDB in plasma. If it was true, central heating of inner-shifted plasma where energy confinement is good would be required for further study. From this point of view, high energy perpendicular NB would play an important role to explore a new regime in LHD. For this sake, an additional new beam line is now planned to be installed in 2010 .

\section{Acknowledgements}

The authors would like to thank the Director-General O. Motojima for his encouragement and support. They are also grateful to contributions from all collaborators in universities and institutions all over the world. Tremendous efforts by the technical staff to operate LHD are greatly acknowledged.
[1] A. Iiyoshi et al., Fusion Technol. 17, 169 (1990).

[2] O. Motojima et al., Nucl. Fusion 47, S668 (2007).

[3] T. Mutoh et al., J. Plasma Fusion Res. 81, 229 (2005).

[4] T. Morisaki et al., Fusion Sci. Technol. 50, 216 (2006).

[5] K. Saito et al., J. Nucl. Mater. 363, 1323 (2007).

[6] K.Y. Watanabe et al., Nucl. Fusion 45, 1247 (2005).

[7] S. Sakakibara et al., Plasma Phys. Control. Fusion 44, A217 (2002).

[8] O. Kaneko et al., Phys. Plasmas 9, 2020 (2002).

[9] N. Ohyabu et al., Phys. Rev. Lett. 97, 05502 (2006).

[10] T. Morisaki et al., Phys. Plasmas 14, 056113 (2007).

[11] R. Sakamoto et al., Plasma Fusion Res. 2, 047 (2007).

[12] R. Sakamoto et al., Nucl. Fusion 41, 381 (2001).

[13] S. Sudo et al., Nucl. Fusion 30, 11 (1990).

[14] J. Miyazawa et al., Nucl. Fusion 48, 015003 (2008).

[15] S. Toda and K. Itoh, Plasma Fusion Res. 3, S1069 (2008).

[16] K. Ida et al., Phys. Rev. Lett. 86, 5297 (2001).

[17] T. Shimozuma et al., Plasma Phys. Control. Fusion 45, 1183 (2003).

[18] Y. Takeiri et al., Fusion Sci. Technol. 46, 106 (2004).

[19] M. Yokoyama et al., Nucl. Fusion 47, 1213 (2007).

[20] Y. Takeiri et al., Nucl. Fusion 47, 1078 (2007).

[21] S. Murakami et al., Fusion Sci. Technol. 46, 241 (2004).

[22] C.D. Beidler and W.D. D'haeseleer, Plasma Phys. Control. Fusion 37, 463 (1995).

[23] K. Ida et al., Nucl. Fusion 45, 391 (2005).

[24] M. Yoshinuma et al., EX/P5-1, $22^{\text {nd }}$ IAEA Fusion Energy Conference, Geneva (2008), submitted to Nucl. Fusion.

[25] K. Tanaka et al., Fusion Sci. Technol. 51, 97 (2007).

[26] K. Ida et al., Phys. Plasmas 16, 056111 (2009). 